\title{
Perceptions par des patients atteints d'arthrite inflammatoire débutante de leur incapacité parentale, de leur stress parental et du comportement de leurs enfants
}

\author{
P. Zelkowitz, D. Éd. (1); K. J. Looper, M.D. (1); S. S. Mustafa, Ph. D. (1); M. Purden, Ph. D. (2); M. Baron, M.D. (3); \\ McGill Early Arthritis Research Group*
}

Cet article a fait l'objet d'une évaluation par les pairs.

Résumé

Introduction : Dans cette étude, nous examinons l'association entre les effets caractéristiques de l'arthrite inflammatoire et les perceptions de patients atteints d'arthrite inflammatoire débutante à propos de leur santé mentale, de leur incapacité parentale, de leur stress parental et du comportement de leurs enfants.

Méthodologie : Le recrutement de patients dont l'arthrite inflammatoire était en phase initiale (survenue depuis plus de 6 semaines et moins de 18 mois) a été effectué à partir d'un registre de cas d'arthrite inflammatoire débutante fournissant des caractéristiques personnelles ainsi que des mesures de la douleur, du fonctionnement physique et de l'activité de la maladie. Les patients ont auto-évalué leur incapacité parentale, leur stress parental, leur tendance à la dépression et les problèmes de comportement de leurs enfants à l'aide, respectivement, de l'Indice d'incapacité parentale, de l'Indice de stress parental, du questionnaire Center for Epidemiologic Studies - Depression Mood Scale [Échelle d'évaluation de l'état dépressif et de l'humeur du Center for Epidemiologic Studies] et de la Child Behavior Checklist [Liste de contrôle du comportement des enfants].

Résultats : La douleur, le dysfonctionnement physique, le nombre d'articulations douloureuses et l'évaluation médicale globale de l'activité de la maladie étaient associés à l'incapacité parentale. Les mesures autodéclarées de l'incapacité parentale étaient associées à celles de la dépression et du stress parental. Le stress parental était associé à la déclaration de problèmes de comportement d'intériorisation et d'extériorisation de l'enfant, tandis que l'incapacité parentale était associée à la déclaration de problèmes de comportement d'extériorisation de l'enfant.

Conclusion : Cette étude souligne la possibilité d'une relation réciproque entre les aspects physiques de l'activité de la maladie, l'incapacité parentale et la détresse du parent et de l'enfant chez les patients atteints d'arthrite inflammatoire débutante.

Mots-clés :incapacité parentale, stress parental, comportement des enfants, arthrite, fonctionnement physique

\section{Introduction}

L'arthrite regroupe plus d'une centaine d'affections rhumatismales touchant les articulations et les tissus voisins. L'arthrite inflammatoire (AI) est caractérisée par une inflammation des articulations en raison de perturbations du système immunitaire. C'est une affection invalidante et douloureuse, associée à une dégradation $\mathrm{du}$ fonctionnement psychologique et social $^{1,2}$. La polyarthrite rhumatoïde (PR), la forme la plus répandue de l'arthrite inflammatoire, est caractérisée par une synovite destructrice chronique. La forme non différenciée de l'arthrite, qui ne satisfait pas aux critères de classification de la maladie, peut soit se résoudre, soit évoluer vers une arthrite rhumatoïde complète.

Une maladie physique touchant un parent peut avoir diverses répercussions sur ses enfants. Le parent peut être incapable de s'acquitter des tâches courantes de soins aux enfants et, de ce fait, demander à ces derniers d'assumer de nouvelles responsabilités domestiques ${ }^{3}$. De plus, le parent peut ne pas être disponible affectivement à cause de la douleur, de la fatigue et des préoccupations qu'engendre chez lui la maladie $^{4,5}$. Le stress familial associé à la perte possible de revenu ou aux conflits conjugaux au sujet de l'évolution des rôles

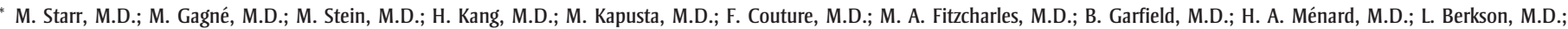
C. Pineau, M.D.; A. Gutkowski, M.D.; M. Zummer, M.D.; J. P. Mathieu, M.D.; S. Mercille, M.D.; S. Ligier, M.D.; J. Krasny, M.D.; C. Bertrand, M.D.; S. Y. Yuen, M.D.; J. Schulz, M.D.

Rattachement des auteurs :

1. Département de psychiatrie, Université McGill, Montréal (Québec), Canada

2. École de sciences infirmières, Université McGill, Montréal (Québec), Canada

3. Département de rhumatologie, Université McGill, Montréal (Québec), Canada

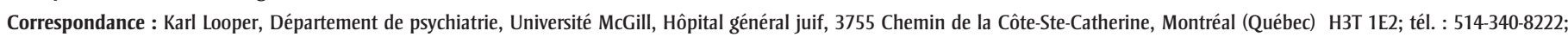
téléc. : 514-230-8126; courriel : karl.looper@mcgill.ca
} 
et de la répartition des tâches peut également nuire à la relation parent-enfant ${ }^{6}$. L'enfant aura davantage de difficulté à s'adapter à la situation si le parent malade manifeste de la détresse psychologique ${ }^{4}$ et si son comportement parental en souffre ${ }^{5}$.

Les rares études effectuées auprès de familles dont un des parents souffre d'arthrite ont mis en lumière d'importants effets négatifs. Ainsi, dans une étude exploratoire, Grant et collab. ${ }^{7}$ ont montré que les parents et les grands-parents atteints de PR éprouvent des difficultés à accomplir des tâches fondamentales liées aux soins des enfants, par exemple soulever un enfant. Katz et collab. ${ }^{8}$ ont observé que les femmes atteintes de PR sont incapables d'exercer certaines activités parentales et, par conséquent, assument moins de fonctions à cet égard. Backman et collab. ${ }^{9}$ ont effectué une étude qualitative de la situation de mères atteintes d'arthrite et ont conclu que les conséquences tant positives que négatives de l'arthrite inflammatoire sur la maternité étaient considérables. Ils ont remarqué que l'exercice du rôle maternel fluctuait en fonction du soutien disponible et de l'équilibre imprévisible entre fatigue et énergie9 ${ }^{9}$ Par conséquent, la cohésion familiale s'en trouvait accrue à certains moments, tandis qu'à d'autres, la famille éprouvait du regret de ne pouvoir s'adonner à certaines activités ${ }^{9}$.

Les patients arthritiques ont souvent l'impression de ne pas fonctionner correctement avec leurs enfants et petitsenfants $^{10}$. Les auteurs d'une étude ont signalé que les enfants de patients atteints de PR avaient davantage de tracas quotidiens, des réseaux de soutien social moins étendus et plus de problèmes d'ajustement social que ceux du groupe témoin ${ }^{11}$. Les enfants adolescents dont un parent était atteint de PR ont fait état d'une estime de soi inférieure à celle de leurs homologues dont les parents étaient en bonne santé ${ }^{12}$.

L'essentiel des recherches effectuées dans ce domaine est constitué de petites études qualitatives. Celles-ci portent souvent sur des patients qui sont malades depuis de nombreuses années. Toutefois, lorsque la maladie en est à ses premiers stades, les problèmes parentaux peuvent être différents de ceux qui se posent lorsque l'arthrite inflammatoire est installée depuis longtemps. Il convient donc d'effectuer des recherches examinant le lien entre le rôle parental et les enfants, la santé mentale et les effets de la maladie dès le début de sa trajectoire, de façon à élaborer des interventions thérapeutiques visant des facteurs particuliers dans la phase initiale de la maladie, afin d'améliorer ultérieurement l'état de santé et l'exercice du rôle parental.

Nous avons donc entrepris cette étude dans le but d'explorer les associations entre les variables de la maladie (douleur, dysfonctionnement physique et activité de la maladie), la souffrance mentale, les facteurs parentaux (capacité parentale et stress parental) et les mesures du comportement des enfants durant les premiers 18 mois après l'apparition de l'arthrite inflammatoire chez le parent.

\section{Méthodologie}

\section{Participants}

Deux cent cinquante-sept patients ont été inscrits dans le McGill Early Arthritis Registry (McEAR) [registre de McGill des cas d'arthrite inflammatoire débutante] entre mars 2006 et mai 2009. Les patients avaient été dirigés vers le McEAR par 21 rhumatologues travaillant à Montréal (Québec). Les rhumatologues participants, qui travaillent tous en cabinet privé ou dans une clinique de soins ambulatoires, avaient été invités à recruter tous les nouveaux patients atteints d'arthrite inflammatoire débutante (AID) qui satisfaisaient aux critères d'inclusion, c'est-àdire avoir reçu récemment un diagnostic d'arthrite inflammatoire, caractérisée par la présence d'une inflammation touchant au moins une articulation depuis au moins 6 semaines et au plus 18 mois. (On a évalué la durée de la maladie en demandant au patient «Quand cet épisode de douleur articulaire a-t-il commencé? » et " avez-vous déjà connu des épisodes comparables d'articulations douloureuses et enflées avant celui-ci?»). Les patients étaient âgés de 18 ans ou plus, parlaient anglais ou français, et ont accepté de se prêter à des examens physiques et à des analyses en laboratoire à intervalles réguliers, ainsi que de répondre à des questionnaires visant à consigner leurs caractéristiques personnelles et à évaluer l'incapacité, la douleur et les facteurs psychosociaux reliés à leur maladie. Les principaux critères d'exclusion étaient des signes cliniques de lésion articulaire ancienne évoquant un épisode antérieur de $P R$, un diagnostic d'affection rhumatismale autre que de PR ou d'arthrite inflammatoire non différenciée, une limitation fonctionnelle sévère imputable à une maladie autre que l'arthrite, et toute affection empêchant de donner un consentement éclairé.

Sur les 257 patients inscrits au McEAR, 80 avaient des enfants âgés de moins de 18 ans qui vivaient avec eux. Ces 257 patients inscrits au McEAR ont tous été invités à participer à des études psychosociales avec visite à domicile des intervieweurs, et 104 (40,5 \%) ont accepté. Parmi les 104 participants, 29 avaient des enfants âgés de moins de 18 ans qui vivaient avec eux. Ces 29 parents participants ont accepté de prendre part à l'étude et ont constitué l'échantillon de celle-ci, ce qui donne un taux de réponse de $36 \%$ (29 des 80 patients inscrits au McEAR ayant des enfants de moins de 18 ans) si l'on considère le McEAR dans son ensemble, et de $100 \%$ par rapport à ceux qui avaient accepté de prendre part à l'étude psychosociale.

Tous les patients inscrits au registre ont été vus par une des deux infirmières de l'équipe de recherche, la rencontre ayant eu lieu au cabinet de leur rhumatologue traitant, lequel restait responsable de leurs soins cliniques. (Cet arrangement visait à rendre plus facile la participation des patients vivant sur l'île de Montréal ou dans ses environs.) Chaque infirmière avait reçu une formation pour effectuer un dénombrement complet des articulations douloureuses et enflées. Les infirmières, les médecins et les patients étaient au courant des évaluations faites par les autres. 
Un intervieweur expérimenté a rencontré chaque patient à son domicile dans les 10 jours suivant la visite d'inscription à l'étude; il ignorait la teneur des renseignements sur les patients consignés dans la base de données du registre principal. L'intervieweur a obtenu le consentement des patients avant de procéder à l'entrevue et les a aidés à remplir les questionnaires de l'étude. Les patients recevaient 25 \$ pour chaque rencontre avec l'intervieweur. Tous les patients inscrits au McEAR ont signé un formulaire de consentement éclairé et l'étude a été autorisée par les comités d'éthique de la recherche de l'université McGill et de l'Hôpital général juif.

\section{Mesures des effets de la maladie}

\section{Fonctionnement physique}

Nous avons mesuré le fonctionnement physique à l'aide du Medical Outcomes Study Short-Form 36 (SF-36) ${ }^{13}$ qui, grâce à ses bonnes propriétés psychométriques, est fréquemment utilisé pour évaluer la qualité de vie liée à la santé. Il se décompose en huit dimensions : fonctionnement physique, fonctionnement social, limitations des rôles dues aux problèmes physiques, limitations des rôles dues aux problèmes émotionnels, santé mentale, vitalité, douleurs physiques et perception de la santé en général. Les scores varient de 0 (pire) à 100 (meilleur). Les huit échelles sont combinées en deux mesures sommaires dont l'une, le score de la composante physique (SF36-PCS), donne une estimation globale de la santé physique, mais nous avons plutôt choisi de mesurer la fonction physique directement à l'aide du score de la dimension du fonctionnement physique. Nous avons mesuré la douleur de manière plus précise à l'aide du Questionnaire McGill sur la douleur (QMD) ${ }^{14}$.

\section{Douleur}

La douleur a été évaluée à l'aide de la forme abrégée du $\mathrm{QMD}^{15}$, qui contient 11 éléments portant sur la dimension sensorielle de la douleur et quatre autres concernant sa dimension affective. Chaque descripteur est classé selon une échelle d'intensité à quatre degrés (0-3; aucune à sévère), et les scores totaux varient de 0 (aucune douleur) à 45 (douleur sévère). Le QMD est fréquemment utilisé et possède de bonnes propriétés psychométriques. Dans cette étude, nous avons utilisé le score total associé à la douleur.

\section{Activité de la maladie}

Nous avons évalué l'activité de la maladie à partir du nombre d'articulations enflées et douloureuses, qui a été déterminé selon la méthode de l'American College of Rheumatology ${ }^{16}$. Nous avons également mesuré la concentration sanguine d'un réactif de phase aiguë, la protéine C-réactive (PCR). La combinaison du nombre d'articulations enflées, du nombre d'articulations douloureuses, de l'évaluation globale de l'activité de la maladie chez le patient et de la concentration sanguine de PCR donne le Disease Activity Score in 28 joints (DAS28) (score d'activité de la maladie pour 28 articulations $)^{17-20}$. Le DAS28, fondé sur 28 articulations constituant un sous-ensemble des critères de l'American College of Rheumatology, a été élaboré ${ }^{17}$ et validé ${ }^{18}$ pour les patients atteints de PR et est considéré comme une mesure valide de l'activité de la maladie ${ }^{18}$. Les critères de validation incluent les corrélations avec un groupe choisi d'autres variables de la maladie (validité corrélationnelle), avec l'incapacité physique et l'avis d'un groupe de rhumatologues cliniciens (validité de critère respectivement I et II), ainsi qu'avec l'atteinte des mains et des pieds évaluée par radiographie (validité de

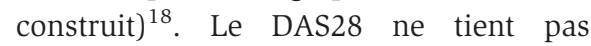
compte des articulations de la cheville et du pied ${ }^{21}$.

Dans notre étude, nous avons procédé à l'évaluation globale de l'activité de la maladie par le patient à l'aide d'une échelle visuelle analogique à 11 degrés allant de 0 (meilleur résultat) à 10 (pire résultat) par rapport à la semaine précédente. Le rhumatologue a coté l'évaluation globale médicale de l'activité de la maladie effectuée à l'aide d'une échelle d'évaluation numérique à 11 degrés allant de 0 (aucune activité arthritique) à 10 (arthrite la plus sévère).

\section{Mesures psychosociales et mesures des compétences parentales et du fonctionnement des enfants}

\section{Center for Epidemiologic Studies - Depression Mood Scale}

Le Center for Epidemiologic Studies Depression Mood Scale (CES-D) [Échelle d'évaluation de l'état dépressif et de l'humeur du Center for Epidemiologic Studies] est un questionnaire d'autoévaluation de 20 éléments destiné à mesurer la dépression dans la population génér$\mathrm{ale}^{22}$. Le répondant doit indiquer à quelle fréquence chaque expérience décrite dans l'élément a été ressentie au cours de la semaine précédente. Les scores vont de 0 à 60, un score plus élevé indiquant une dépression plus profonde. Un score de coupure de 16 est la norme pour repérer une dépression, mais, dans les maladies chroniques comme la $\mathrm{PR}$, on préconise plutôt un score de coupure de $19^{23,24}$. Dans notre étude, nous avons utilisé le score total (variable continue) pour mesurer la sévérité des symptômes.

\section{Indice de stress parental}

L'Indice de stress parental (ISP) (version abrégée du questionnaire) ${ }^{25}$ est un instrument fondé sur l'autodéclaration, constitué de 36 éléments et qui évalue la détresse parentale. Il est constitué de trois sous-échelles, Détresse parentale, Interaction parent-enfant dysfonctionnelle et Enfant difficile. Chaque élément est évalué sur une échelle de 1 ("pas du tout d'accord ») à 5 (" tout à fait d'accord »). Plus le score est élevé, plus le stress est grand. Le coefficient $\alpha$ de Cronbach varie de 0,88 à 0,95 et on a établi la validité de construit de l'échelle par rapport aux mesures des problèmes de comportement des enfants ${ }^{25}$. Dans cette étude, c'est le score total qui a été utilisé.

\section{Indice d'incapacité parentale}

L'Indice d'incapacité parentale (IIP) a été élaboré pour mesurer la fonction et l'incapacité parentales et a été validé chez les femmes atteintes de $\mathrm{PR}^{8}$. Les 27 éléments de cette échelle sont notés de 0 à 3,0 signifiant aucune incapacité et 3 indiquant l'incapacité à accomplir les tâches parentales. L'IIP est le niveau de 
difficulté moyen mesuré dans les différentes dimensions correspondant à la cohorte du parent et au groupe d'âge de l'enfant. Deux scores sommaires ont été élaborés, celui employé dans cette étude étant l'IIP modifié (IIPM) ${ }^{8}$. Cette échelle a été validée dans une population atteinte de PR.

\section{Child Behavior Checklist}

Les versions adaptées aux enfants âgés de 1,5 à 5 ans et de 6 à 18 ans de la Child Behavior Checklist $(\mathrm{CBCL})^{26,27}$ [Liste de contrôle du comportement des enfants] ont été utilisées pour évaluer les problèmes de comportement des enfants. Les parents évaluent le comportement de leurs enfants au cours des deux derniers mois. À chaque élément est attribué un score allant de 0 pour " pas vrai » à 2 pour « très ou souvent vrai ». Les deux versions possèdent trois scores à "large bande " (scores sommaires) portant sur les problèmes d'intériorisation, les problèmes d'extériorisation et les problèmes totaux. Les problèmes d'intériorisation mesurés sont l'anxiété, la dépression et les symptômes somatiques; les problèmes d'extériorisation mesurés sont les problèmes de conduite, l'hyperactivité et l'agressivité. La fiabilité de test-retest varie de 0,88 à 0,91 pour les échelles à " large bande », et la fiabilité interévaluateurs de la CBCL est de 0,72 pour les problèmes d'intériorisation et de 0,85 pour les problèmes d'extériorisation $^{26}$. Nous avons utilisé les scores d'intériorisation et d'extériorisation pour les deux groupes d'âge. Pour obtenir des données comparables, lorsque deux enfants ou plus vivaient à la maison, les parents ont été invités à évaluer le comportement de celui des enfants dont l'âge se rapprochait le plus de 10 ans.

\section{Analyse statistique}

En raison de la petite taille de l'échantillon ( $n=29$ ), on doit considérer les analyses de données effectuées dans cette étude comme de nature exploratoire. Les données ont été analysées à l'aide du logiciel SPSS version 17 pour Windows (IBM, Chicago, Illinois, États-Unis). On a eu recours à des statistiques descriptives pour résumer les caractéristiques de départ des patients atteints d'AID. On a utilisé des corrélations de Pearson pour calculer les associations entre les différentes variables continues de l'étude. Les données manquantes n'ont pas fait l'objet d'une imputation et nous n'avons pas inclus dans nos calculs les cas pour lesquels manquaient certaines données.

\section{Résultats}

L'âge moyen des participants de l'étude était de près de 42 ans; 20 (69 \%) étaient des femmes et 23 (79 \%) vivaient en couple. Au total, 21 participants (72 \%) avaient un emploi : 14 travaillaient à plein temps, 3 étaient en congé de maladie, 2 travaillaient à temps partiel et 2 étaient travailleurs autonomes. Le revenu annuel total du ménage de plus de la moitié des participants était supérieur à 60000 \$. L'échantillon affichait un niveau de scolarité très élevé, 20 (69 \%) participants ayant un diplôme collégial ou universitaire de premier cycle ou des cycles supérieurs. Sur nos 29 participants, 15 (52 \%) avaient 1 enfant, 10 (35\%) avaient 2 enfants et 4 (14\%) avaient trois enfants âgés de moins de 18 ans vivant à la maison. Chez nos participants, la durée moyenne de la maladie était de 8,24 mois (écart-type : 3,65). Quatre participants (14\%) ont obtenu un score supérieur au point de coupure de 19 au CES-D (voir tableau 1).

L'âge moyen de l'enfant cible (celui dont l'âge se rapprochait le plus de 10 ans) était de 10,6 ans. La proportion de garçons et de filles était presque également distribuée dans l'échantillon, et $48 \%$ des enfants cibles étaient des filles. Trois enfants ont obtenu des scores supérieurs à la valeur de coupure clinique de 60 pour l'évaluation des problèmes d'intériorisation et d'extériorisation avec la CBCL.

Le stress parental (ISP) n'était significativement corrélé qu'au score total de l'humeur dépressive du CES-D. L’incapacité parentale autoperçue (IIPM) était, elle, corrélée, outre à l'humeur dépressive (CES-D), à toutes les mesures de la maladie : évaluation médicale globale de l'activité de la maladie, nombre d'articulations douloureuses, dysfonctionnement physique (SF-36) et douleur (QMD). Les problèmes de comportement d'intériorisation et d'extériorisation des enfants perçus par les patients (CBCL, tous les âges) étaient significativement corrélés à l'évaluation médicale globale de l'activité de la maladie. Les symptômes de dépression des parents n'étaient pas associés aux problèmes de comportement de leur enfant. Le tableau 2 indique les relations entre la santé physique et mentale du parent et les problèmes de rôle parental et de comportement de l'enfant.

Le stress parental (ISP) était également significativement corrélé à l'incapacité parentale (IIPM) et aux problèmes d'intériorisation et d'extériorisation des enfants (CBCL). L'incapacité parentale autoperçue (IIPM) était significativement corrélée, outre au stress parental, aux problèmes d'extériorisation de l'enfant (CBCL). Le tableau 3 indique les interrelations entre les variables du rôle parental et celles du comportement des enfants.

D’autres variables liées à la maladie, dont le nombre d'articulations enflées, la concentration de PCR et le score au DAS28, ne présentaient pas de corrélation significative avec l'une ou l'autre des mesures du rôle parental ou du comportement des enfants.

\section{Analyse}

Les tâches parentales sont extrêmement exigeantes sur le plan tant physique qu'émotionnel $^{28}$. Compte tenu de la rareté des articles scientifiques sur la manière dont les patients atteints d'arthrite chronique qui ont de jeunes enfants gèrent tant leur maladie que leurs tâches parentales, nous avons cherché à examiner la relation entre l'arthrite dans ses premiers stades (entre 6 semaines et 18 mois) et la détresse autoévaluée par le patient, son aptitude à s'acquitter de ses tâches parentales et sa perception du comportement de son enfant.

Selon les principales constations de cette étude, l'arthrite, même dans ses premiers stades, interfère bel et bien avec le rôle parental. Nos patients ont souffert d'une incapacité parentale croissante au fur et à mesure de l'augmentation de la valeur des multiples mesures de l'activité de la maladie, notamment la douleur, le dysfonctionnement physique, le nombre 
TABLEAU 1

Caractéristiques des participants de l'étude $(\mathrm{N}=29)$

\begin{tabular}{|c|c|c|c|}
\hline Variable de l'étude & $\begin{array}{l}\text { Fréquence } \mathbf{n} \\
(\%)\end{array}$ & $\begin{array}{l}\text { Moyenne } \\
\text { ('́T) }\end{array}$ & $\begin{array}{l}\text { Intervalle de } \\
\text { confiance à } \\
\quad 95 \%\end{array}$ \\
\hline \multicolumn{4}{|l|}{ Caractéristiques des participants } \\
\hline Âge (années) & & $41,97(7,95)$ & 26 à 57 \\
\hline Femme & $20(69)$ & & \\
\hline En couple & $23(79)$ & & \\
\hline Occupant un emploi & $21(72)$ & & \\
\hline \multicolumn{4}{|l|}{ Revenu annuel du ménage } \\
\hline inférieur à 60000 \$ & $11(38)$ & & \\
\hline $60000 \$$ et plus & $17(59)$ & & \\
\hline \multicolumn{4}{|l|}{ Niveau de scolarité } \\
\hline Secondaire ou moins & $9(31)$ & & \\
\hline Collège ou plus & $20(69)$ & & \\
\hline \multicolumn{4}{|l|}{ Nombre d'enfants } \\
\hline 1 & $15(52)$ & & \\
\hline 2 & $10(35)$ & & \\
\hline 3 & $4(14)$ & & \\
\hline Âge de l'enfant cible & & $10,60(5,10)$ & 1,00 à 18,00 \\
\hline Sexe de l'enfant cible ${ }^{\mathrm{a}}$ : fille & $13(48)$ & & \\
\hline \multicolumn{4}{|l|}{ Caractéristiques de la maladie } \\
\hline Durée (mois) & & $8,24(3,65)$ & 4,00 à 18,00 \\
\hline Fonctionnement physique SF-36 & & $60,04(28,90)$ & 5,00 à 100,00 \\
\hline Douleur totale QMD & & $8,60(11,17)$ & 0,00 à 45,00 \\
\hline Nombre d'articulations enflées & & $9,19(9,56)$ & 0,00 à 39,00 \\
\hline Nombre d'articulations douloureuses & & $16,00(12,76)$ & 0,00 à 43,00 \\
\hline PCR (mg/L) & & $22,87(22,49)$ & 0,30 à 69,00 \\
\hline DAS28 & & $5,30(1,80)$ & 2,61 à 8,08 \\
\hline Évaluation médicale globale de l'activité de la maladie & & $3,96(2,71)$ & 0,00 à 10,00 \\
\hline \multicolumn{4}{|l|}{ Variables psychosociales } \\
\hline Humeur dépressive totale CES-D & & $10,03(10,58)$ & 0,00 à 41,00 \\
\hline ISP & & $63,89(19,43)$ & 36,00 à 100,00 \\
\hline IIPM tous les âges & & $0,65(0,61)$ & 0,00 à 1,95 \\
\hline Problèmes d'extériorisation, tous les âges, $\mathrm{CBCL}$ & & $47,96(8,18)$ & 34,00 à 65,00 \\
\hline Problèmes d'intériorisation, tous les âges, $\mathrm{CBCL}$ & & $50,55(11,39)$ & 33,00 à 78,00 \\
\hline
\end{tabular}

Abréviations : CBCL, Child Behavior Checklist [Liste de contrôle du comportement des enfants]; CES-D, Center for Epidemiologic Studies - Depression Mood Scale; DAS28, Disease Activity Score in 28 joints [Score d'activité de la maladie au niveau de 28 articulations]; ÉT, écart-type; IIPM, Indice d'incapacité parentale modifié; ISP, Indice de stress parental; PCR, protéine C-réactive; QMD, Questionnaire McGill sur la douleur; SF-36, Medical Outcomes Study Short Form 36.

a Pour obtenir des données comparables, s'il y avait deux enfants ou plus vivant à la maison, les parents étaient invités à évaluer le comportement de celui dont l'âge était le plus proche de 10 ans.

d'articulations douloureuses et l'évaluation médicale globale de l'activité de la maladie. Les patients ont fait état de difficultés pour, notamment, se pencher, avoir des activités extérieures ou recevoir d'autres enfants à la maison. Cette impression de ne pas s'acquitter adéquatement de ses tâches parentales peut avoir engendré un sentiment de détresse, comme semblent l'indiquer l'étroite corrélation observée entre l'incapacité parentale et le stress parental et le fait que ces deux facteurs soient liés au score obtenu à l'évaluation de l'humeur dépressive réalisée avec le questionnaire CES-D.

Ces observations sont en accord avec les résultats des recherches antérieures portant sur des patients atteints de PR à un stade plus avancé ${ }^{7,8}$. Dans ces études, on a observé que de nombreux patients avaient de la difficulté à s'occuper de leurs enfants et, en particulier, à accomplir les tâches nécessitant un effort physique comme soulever un enfant du sol, ou encore suivre le rythme de leurs enfants. Ces difficultés sont souvent reliées à des problèmes physiques ou psychologiques tels que l'anxiété, la dépression et la culpabilité. White et collab. ${ }^{29}$ ont également constaté que chez les mères souffrant de PR, une plus grande fatigue était un prédicteur significatif d'une fréquence et d'une intensité accrues de problèmes quotidiens dans l'exécution des tâches parentales et d'une plus grande difficulté à surveiller les déplacements de leurs enfants ${ }^{29}$. Les mères atteintes éprouvaient plus de difficultés à surveiller leur enfant si elles étaient plus déprimées et étaient aux prises avec une exacerbation ${ }^{29}$. Nos résultats sont également en accord avec ceux d'une étude très récente ${ }^{30}$ portant sur les répercussions du lupus érythémateux systémique sur la capacité des mères de s'occuper de leurs enfants. Les auteurs ont observé qu'une augmentation de la fatigue et de l'incapacité fonctionnelle se traduisait par des scores d'IIP plus élevés chez les mères ayant des enfants âgés de moins de 18 ans vivant à la maison. La durée moyenne de la maladie de ces mères était de 7 ans. Dans notre étude, nous avons pu déceler une association entre l'incapacité parentale perçue par le patient et les variables de la maladie dans les huit mois suivant, en moyenne, la survenue de cette dernière.

La diminution de l'efficacité du patient dans l'exécution de ses tâches parentales et la détresse psychologique concomitante affectent ses enfants. Les enfants dont les parents présentaient une maladie plus active selon l'évaluation médicale étaient perçus par ces derniers comme présentant davantage de problèmes comportementaux d'intériorisation et d'extériorisation. Le stress parental était associé à des problèmes d'intériorisation et d'extériorisation, tandis que l'incapacité parentale n'était corrélée qu'avec les problèmes d'extériorisation. Il est possible que les enfants que les parents supervisent moins et dont ils s'occupent moins soient plus 
TABLEAU 2

Corrélations produit-moment de Pearson ( $r$ ) entre les variables de l'étude

\begin{tabular}{|c|c|c|c|c|c|c|}
\hline & \multirow[t]{2}{*}{ Statistiques } & \multicolumn{5}{|c|}{ Mesure de la santé/maladie parentale } \\
\hline & & $\begin{array}{l}\text { Humeur dépres- } \\
\text { sive totale CES-D }\end{array}$ & $\begin{array}{l}\text { Évaluation médicale globale } \\
\text { de l'activité de la maladie }\end{array}$ & $\begin{array}{c}\text { Nombre d'articulations } \\
\text { douloureuses }\end{array}$ & $\begin{array}{l}\text { Fonctionnement } \\
\text { physique SF-36 }\end{array}$ & $\begin{array}{l}\text { Douleur } \\
\text { totale } \mathbf{Q M D}^{\mathrm{a}}\end{array}$ \\
\hline \multicolumn{7}{|c|}{ Variables du rôle parental $^{a}$} \\
\hline \multirow{3}{*}{$\begin{array}{l}\text { Indice de stress paren- } \\
\text { tal (ISP) }\end{array}$} & $r$ & $0,565^{*}$ & 0,360 & 0,039 & $-0,149$ & 0,045 \\
\hline & $p$ & 0,002 & 0,077 & 0,874 & 0,488 & 0,834 \\
\hline & $n$ & 27 & 25 & 19 & 24 & 24 \\
\hline \multirow{2}{*}{$\begin{array}{l}\text { Indice d'incapacité par- } \\
\text { entale (IIPM) }\end{array}$} & $p$ & $<0,001$ & 0,001 & 0,025 & 0,003 & 0,038 \\
\hline & $n$ & 24 & 22 & 17 & 21 & 21 \\
\hline \multicolumn{7}{|c|}{ Problèmes de comportement des enfants $(\mathrm{CBCL})^{\mathrm{a}}$} \\
\hline Intériorisation & $r$ & 0,348 & $0,503^{*}$ & 0,017 & $-0,148$ & $-0,020$ \\
\hline \multirow{2}{*}{ Extériorisation } & $p$ & 0,074 & 0,035 & 0,950 & 0,218 & 0,684 \\
\hline & $n$ & 25 & 23 & 18 & 22 & 22 \\
\hline
\end{tabular}

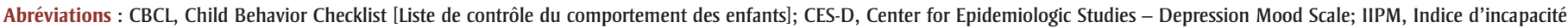
parentale modifié; ISP, Indice de stress parental; QMD, Questionnaire McGill sur la douleur; SF-36, Medical Outcomes Study Short Form 36; ÉT, écart-type.

${ }^{a}$ Ces données sont fondées sur les perceptions des patients.

* Corrélation significative à un niveau de 0,05 (bilatéral).

** Corrélation significative à un niveau de 0,01 (bilatéral).

susceptibles de désobéir et d'être agressifs. Dans une étude réalisée par Welch et collab. ${ }^{31}$, les parents qui avaient reçu depuis peu un diagnostic de cancer n'ont pas décelé de problèmes affectifs ou comportementaux chez leurs enfants à l'aide de l'échelle CBCL; toutefois ces derniers, et en particulier les adolescentes, ont fait état de symptômes d'anxiété et de dépression et d'un comportement agressif $^{31}$. Au vu de ces constatations, les études à venir devraient porter sur les
TABLEAU 3

Corrélations produit-moment de Pearson $(r)$ entre les variables du rôle parental et celles du comportement des enfants

\begin{tabular}{|c|c|c|c|c|c|}
\hline & \multirow[t]{2}{*}{ Statistiques } & \multicolumn{2}{|c|}{$\begin{array}{c}\text { Variables des } \\
\text { compétences } \\
\text { parentales }\end{array}$} & \multicolumn{2}{|c|}{ Variables du comportement des enfants } \\
\hline & & ISP & IIPM & $\mathrm{CBCL}$ - intériorisation & CBCL - extériorisation \\
\hline \multirow[t]{3}{*}{ ISP } & $r$ & - & $0,441^{*}$ & $0,537^{*}$ & 0,572 \\
\hline & $p$ & - & 0,031 & 0,010 & 0,003 \\
\hline & $n$ & - & 24 & 22 & 25 \\
\hline \multirow[t]{3}{*}{ IIPM } & $r$ & - & - & 0,232 & $0,486^{*}$ \\
\hline & $p$ & - & - & 0,325 & 0,022 \\
\hline & $n$ & - & - & 20 & 22 \\
\hline \multirow{3}{*}{$\begin{array}{l}\text { Problèmes de comporte- } \\
\text { ment des enfants (CBCL) } \\
\text {-intériorisation }\end{array}$} & $r$ & - & - & - & $0,759^{* *}$ \\
\hline & $p$ & - & - & - & 0,000 \\
\hline & $n$ & - & - & - & 22 \\
\hline
\end{tabular}

Abréviations : CBCL, Child Behavior Checklist; IIPM, Indice d'incapacité parentale modifié; ISP, Indice de stress parental.

* Corrélation significative à un niveau de 0,05 (bilatéral).

** Corrélation significative à un niveau de 0,01 (bilatéral). autodéclarations non seulement des parents, mais aussi des enfants, afin de permettre de brosser un portrait plus complet de la détresse des enfants face à la maladie chronique d'un parent.

Le fait que ce soit l'évaluation médicale globale de l'activité de la maladie, et non pas les mesures du fonctionnement, de la douleur ou du nombre d'articulations atteintes, qui soit associée aux perceptions par les parents de comportements d'intériorisation et d'extériorisation de leurs enfants pourrait indiquer que la détresse du patient face à ses responsabilités parentales influe dans une certaine mesure sur l'évaluation médicale globale. Les rhumatologues pourraient être invités à adresser leur patient à des professionnels de la santé dans des domaines connexes pour obtenir au besoin une évaluation complémentaire.

\section{Limites}

Notre étude a été limitée par la faible taille de son échantillon $(n=29)$. Seuls $30 \%$ des participants inscrits au registre McEAR 
avaient des enfants âgés de moins de 18 ans vivant à la maison. Notre étude portant sur les compétences parentales, nous étions nécessairement limités aux patients ayant des enfants vivant avec eux à la maison. Nous ne disposions donc pas d'une puissance suffisante pour déceler des associations significatives entre les variables de l'étude. Par exemple, il n'a pas été possible d'explorer les différences sur le plan de l'emploi ou d'autres responsabilités non professionnelles qui pourraient avoir eu une influence sur le stress parental et l'humeur. Néanmoins, nous avons pu mettre en évidence des relations significatives entre l'humeur du parent et la manière dont il assume son rôle parental, ainsi qu'entre l'activité de la maladie et le comportement parental.

Comme il s'agissait d'une étude transversale, il n'a pas été possible d'estimer la relation de cause à effet dans les associations observées. Il faudrait effectuer des études longitudinales pour évaluer comment la progression de la maladie peut influer sur la relation parent-enfant et sur les enfants. Toutefois, d'après les recherches antérieures selon lesquelles, d'une part, l'incapacité d'accomplir les tâches quotidiennes auxquelles on accorde de l'importance est un puissant prédicteur de l'apparition subséquente de symptômes dépressifs, et d'autre part les symptômes dépressifs conduisent à une diminution du fonctionnement parental ${ }^{32}$, nous pensons que l'incapacité à accomplir ses tâches parentales pourrait être associée à une détresse psychologique, laquelle aurait des répercussions négatives sur les enfants.

Une troisième limite de notre étude est le recours à l'autodéclaration par les parents de leurs symptômes psychologiques et physiques et des effets sur les enfants, ce qui pourrait engendrer une variance due à une méthode commune. Les recherches futures devraient suivre les familles de manière longitudinale afin de voir comment la progression de la maladie ou sa régression après un traitement influe sur la relation parent-enfant et sur les enfants. De plus, il serait utile d'obtenir de l'information auprès des enfants euxmêmes et de demander des évaluations indépendantes faites par des enseignants ou d'autres informateurs au fait de la situation.

Enfin, d'après des études récentes, l'échelle DAS28 pourrait sous-estimer l'activité de la maladie chez certains patients atteints de PR dont la maladie s'est déclarée principalement dans les articulations du pied, et plus particulièrement durant les deux premières années de la maladie ${ }^{21}$.

\section{Conclusion}

Notre étude a mis en évidence une incapacité potentielle des parents à prodiguer à leurs enfants des soins de qualité en raison de la douleur causée par l'arthrite, $\mathrm{du}$ dysfonctionnement physique et de l'activité de cette maladie. La déficience physique autoperçue était associée à une détresse psychologique chez les parents et à la perception de problèmes de comportement chez leur enfant, ce qui met en lumière l'interdépendance et la complexité des relations entre la santé physique et psychologique du parent et les interactions parent-enfant. Une approche pluridisciplinaire des soins pour les parents atteints d'arthrite s'impose. Il est important de prendre en charge non seulement les symptômes physiques de l'arthrite, mais aussi la détresse affective découlant de la douleur et de la déficience fonctionnelle associées à la maladie. Backman et collab. ${ }^{9}$ ont proposé quelques stratégies susceptibles d'aider les parents atteints d'arthrite à accomplir leurs tâches parentales, comme une réduction des attentes, des approches souples ou alternatives dans l'accomplissement des tâches parentales, des interventions de santé publique et la formulation de conseils crédibles fondés sur l'expérience de parents vivant avec l'arthrite ${ }^{9}$. Il pourrait également être salutaire de reformuler et d'expliquer les comportements liés à cette maladie aux enfants ${ }^{33}$ et d'encourager ces derniers à faire preuve de maturité dans leur comportement ${ }^{34}$.

\section{Remerciements}

Cette étude a été financée par la subvention 8455 du Fonds de la recherche en santé du Québec.

\section{Références}

1. Anderson KO, Bradley LA, Young LD, McDaniel LK, Wise CM. Rheumatoid arthritis: review of psychological factors related to etiology, effects, and treatment. Psychol Bull. 1985;98(2):358-87.

2. Fex E, Larsson BM, Nived K, Eberhardt K. Effect of rheumatoid arthritis on work status and social and leisure time activities in patients followed 8 years from onset. J Rheumatol. 1998;25(1):44-50.

3. Lundwall RA. Parents' perceptions of the impact of their chronic illness or disability on their functioning as parents and on their relationships with their children. Fam J. 2002;10(3):300-7.

4. Korneluk YG, Lee CM. Children's adjustment to parental physical illness. Clin Child Fam Psychol Rev. 1998;1(3):179-93.

5. Sigal JJ, Perry JC, Robbins JM, Gagné MA, Nassif E. Maternal preoccupation and parenting as predictors of emotional and behavioral problems in children of women with breast cancer. J Clin Oncol. 2003; 21(6):1155-60.

6. Rolland JS. Parental illness and disability: a family systems framework. J Fam Ther. 1999;21(3):242-66.

7. Grant MI, Foster NE, Wright CC, Barlow $\mathrm{JH}$, Cullen LA. Being a parent or grandparent with back pain, ankylosing spondylitis or rheumatoid arthritis : a descriptive postal survey. Musculoskeletal Care. 2004;2(1):17-28.

8. Katz PP, Pasch LA, Wong B. Development of an instrument to measure disability in parenting activity among women with rheumatoid arthritis. Arthritis Rheum. 2003;48(4):935-43.

9. Backman CL, Smith L del F, Smith S, Montie PL, Suto M. Experiences of mothers living with inflammatory arthritis. Arthritis Rheum. 2007;57(3):381-8.

10. Barlow JH, Cullen LA, Foster NE, Harrison $\mathrm{K}$, Wade M. Does arthritis influence perceived ability to fulfill a parenting role? Perceptions of mothers, fathers and grandparents. Patient Educ Couns. 1999;37(2): 141-51. 
11. Turner-Cobb JM, Steptoe A, Perry L, Axford J. Adjustment in patients with rheumatoid arthritis and their children. J Rheumatol. 1998;25(3):565-71.

12. Hirsch BJ, Moos RH, Reischl TM. Psychosocial adjustment of adolescent children of a depressed, arthritic, or normal parent. J Abnorm Psychol. 1985;94(2):15464.

13. McHorney CA, Ware JE Jr, Raczek AE. The MOS 36-Item Short-Form Health Survey (SF-36) : II. Psychometric and clinical tests of validity in measuring physical and mental health constructs. Med Care. 1993;31(3):247-63.

14. Melzack R. The McGill Pain Questionnaire: major properties and scoring methods. Pain. 1975;1(3):277-99.

15. Melzack R. The short-form McGill Pain Questionnaire. Pain. 1987;30(2):191-7.

16. Felson DT, Anderson JJ, Boers $M$, Bombardier C, Chernoff M, Fried B et collab. The American College of Rheumatology preliminary core set of disease activity measures for rheumatoid arthritis clinical trials. The Committee on Outcome Measures in Rheumatoid Arthritis Clinical Trials. Arthritis Rheum. 1993; 36(6):729-40.

17. van der Heijde DM, van't Hof MA, van Riel PL, Theunisse LA, Lubberts EW, van Leeuwen MA et collab. Judging disease activity in clinical practice in rheumatoid arthritis: first step in the development of a disease activity score. Ann Rheum Dis. 1990;

49(11):916-20.

18. van der Heijde DM, van't Hof MA, van Riel PL, van Leeuwen MA, van Rijswijk MH, van de Putte LB. Validity of single variables and composite indices for measuring disease activity in rheumatoid arthritis. Ann Rheum Dis. 1992;51(2):177-81.

19. van der Heijde DM, van Riel PL, van Leeuwen MA, van't Hof MA, van Rijswijk $\mathrm{MH}$, van de Putte LB. Prognostic factors for radiographic damage and physical disability in early rheumatoid arthritis. A prospective follow-up study of 147 patients. Br J Rheumatol. 1992;31(8):519-25.
20. Wijnands MJ, van’t Hof MA, van Leeuwen MA, van Rijswijk MH, van de Putte LB, van Riel PL. Long-term second-line treatment : a prospective drug survival study. $\mathrm{Br}$ J Rheumatol. 1992;31(4):253-8.

21. Bakker MF, Jacobs JW, Kruize AA, van der Veen MJ, van Booma-Frankfort C, Vreugdenhil SA et collab. Misclassification of disease activity when assessing individual patients with early rheumatoid arthritis using disease activity indices that do not include joints of feet. Ann Rheum Dis. 2012;71(6):830-5.

22. Radloff LS. The CES-D scale. A self-report depression scale for research in the general population. Appl Psychol Meas. 1977;1(3): 385-401.

23. Martens MP, Parker JC, Smarr KL, Hewett JE, Slaughter JR, Walker SE. Assessment of depression in rheumatoid arthritis : a modified version of the center for epidemiologic studies depression scale. Arthritis Rheum. 2003;49(4):549-55.

24. McQuillan J, Fifield J, Sheehan TJ, Reisine $\mathrm{S}$, Tennen $\mathrm{H}$, Hesselbrock $\mathrm{V}$ et collab. A comparison of self-reports of distress and affective disorder diagnoses in rheumatoid arthritis : a receiver operator characteristic analysis. Arthritis Rheum. 2003;49(3): 368-76.

25. Abidin RR. Parenting stress index: short form (3rd ed: test manual. University of Virginia (36-item version). Odessa (FL) : Psychological Assessment Resources, Inc; 1995.

26. Achenbach TM. Child behavior checklist for ages 6-18. Burlington (VT) : University of Vermont, Research Center for Children, Youth, \& Families; 2001.

27. Achenbach TM, Ruffle TM. The Child Behavior Checklist and related forms for assessing behavioral/emotional problems and competencies. Pediatr Rev. 2000;21(8): 265-71.

28. Umberson D. Relationships with children : explaining parents' psychological wellbeing. J Marriage Fam. 1989;51(4):9991012.

29. White CP, White MB, Fox MA. Maternal fatigue and its relationship to the caregiving environment. Fam Syst Health. 2009; 27(4):325-45.
30. Poole JL, Rymek-Gmytrasiewicz M, Mendelson C, Sanders M, Skipper B. Parenting : the forgotten role of women living with systemic lupus erythematosus. Clin Rheumatol. 2012;31(6):995-1000.

31. Welch AS, Wadsworth ME, Compas BE. Adjustment of children and adolescents to parental cancer. Parents' and children's perspectives. Cancer. 1996;77(7):1409-18.

32. Pakenham KI, Cox S. Test of a model of the effects of parental illness on youth and family functioning. Health Psychol. 2011; à paraître.

33. Allaire S. How a chronically ill mother manages. Am J Nurs. 1988;88(1):46-9.

34. White CP, Mendoza J, White MB, Bond C. Chronically ill mothers experiencing pain : relational coping strategies used while parenting young children. Chronic Illn. 2009;5(1):33-45. 